\title{
Features of steam turbines diagnostics
}

\author{
Ilia Murmanskii ${ }^{1, *}$, Konstantin Aronson ${ }^{1}$, Boris Murmansky ${ }^{1}$, Andrei Sosnovskii ${ }^{1}$, Vladimir Novosyolov ${ }^{1}$, and Yuri Brodov $^{1}$ \\ ${ }^{1}$ Ural Federal University, Turbines \& Engines Dep., 620002, Mira, 19, Ekaterinburg, Russia
}

\begin{abstract}
Enterprises of energy equipment and operational utilities set sights on diagnostic systems. This is necessary for state control and maintenance planning of steam turbines. It is useful for digitalization purposes too. So far, some mathematical systems are already used. Algorithms for flow part, heat expansion system, control system, vibration-based diagnostics and auxiliary equipment are already designed. We designed algorithms just in principle. Adapting them, for the PT-75/80-90 turbine we met with difficulties. Firstly, we should connect them to a single interface. Secondly, adaptation should include features of the equipment, its state (if not new), even operating conditions. Diagnostic signs for each turbine are the most important. We define them based on the operational data. When adapting the algorithms, we reconsider the signs list. We also estimate its coefficients of importance again. This requires experts to study designs, calculations, and modelling. We also analyzed a large amount of operation data at various power plants. To define the state we use tests. Adapting is based on the modes of a specific power station. Following this strategy, we adapt general algorithms for various turbines.
\end{abstract}

\section{Introduction}

At present, a number of power equipment manufacturers and large companies involved in its operation express an increased interest in developing diagnostic systems, for determining the actual condition of equipment and for planning the volumes and terms of its repair, based on this information. Over the last 20-25 years, a number of tasks have been tested of diagnosing steam turbines main and auxiliary equipment at individual power plants [1-9]. For these diagnostic systems, as a rule, the information was supplied by special sensors, and its implementation consisted of process parameters monitoring and of comparing the values of these parameters with standard characteristics. The developed diagnostic algorithms contained the necessary information about defects that occur in the equipment, as well as data for evaluating the manifestations of these defects. However, in most cases, the diagnosis was performed by experts on the basis of the process parameters monitoring data.

\section{Methods and materials}

The main difference between the diagnostic systems currently being created is the possibility of obtaining the information from standard measuring systems of automated process control systems and other (including commercial) information systems of thermal power plants. This allows one to diagnose equipment both in real time and post-operatively, solving, among other things, the forecast tasks. One of the most important issues of introducing diagnostic systems is the need to adapt the diagnosis and condition assessment algorithms developed by experts for specific equipment and its operating conditions. A number of specialists involved in the operation have an opinion about the possibility of extending the algorithms developed for one standard size of equipment to other standard sizes.

Before the diagnostic process, used data should be carefully validated. Three pre-diagnostic stages of validation are suggested for use. Firstly, the data, collected by the automated process control system (APCS), is checked according to availability of the sensor signal. Secondly, each sensor signal has a set range of values, which are determined by the technological process. Thirdly, as the diagnostic is performed not automatically, but by the expert, they have to look through the data by themselves to check if it looks realistic enough. After all, when the diagnostic algorithm is performed, the results are reviewed once again. If the data is too different from the nominal value or the previous one, the expert should check it once again.

Among the algorithms developed for diagnosis, a number of types can be distinguished that differ in the degree of universality (independence from specific conditions) and, on the contrary, in the degree of the diagnosis certainty. The first type of algorithms calculated - implements diagnostic functions in the online mode within the automated process control system or in the postoperative mode. Such computational algorithms include diagnostic algorithms for a steam distribution and turbine regulation systems, for turbine auxiliary equipment (condensing unit, regenerative feed water heating system, hot water heating), for turbine flow path, etc. These algorithms describe the models of

* Corresponding author: i.b.murmansky@urfu.ru 


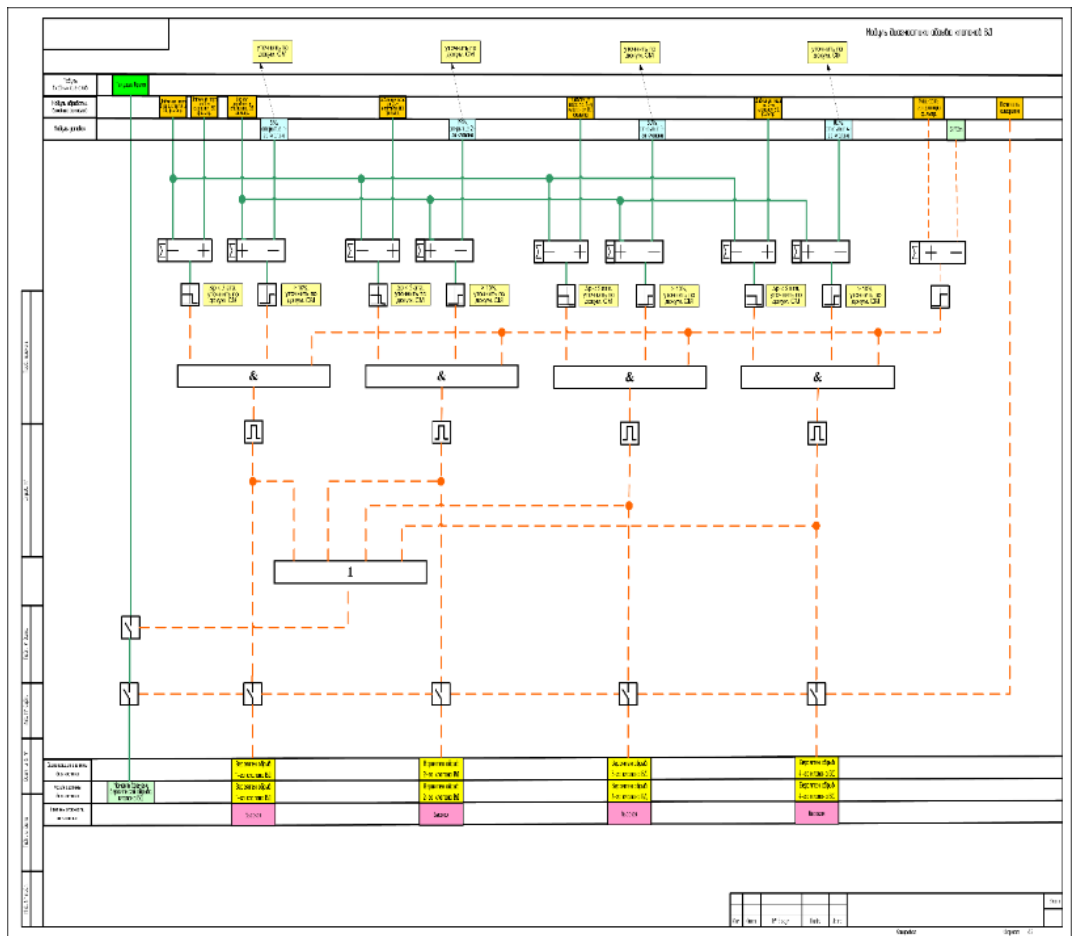

Fig. 1. Diagnostics of high pressure valves stem breakage.

processes occurring in equipment. To the second type of algorithms - the expert one - one can attribute expert systems that analyze, for example, the vibration state of rotating mechanisms, or the system of turbine thermal expansion, and the state of auxiliary equipment. As a rule, such algorithms describe the procedures for determining (evaluating) the signs of defects, and the probability of defects is calculated as the sum of the sign weighting coefficients or by Bayes formula. The third type of algorithms - the digital type - is represented by digital algorithms based on phenomenological relationships between the parameters of the process, the state of the equipment and other indicators, these relationships having been obtained over the test period of equipment operation. This type of algorithm is least related to the type of equipment, its features, and operating modes.

The need to adapt diagnostic systems to other kinds of equipment consists not only in the development of new diagnostic algorithms, but also in the formalization of discourse. This refers to the choice and justification of the normative state (prototype) [10], that is, to a certain model state, in comparison with which diagnostic signs are formed, as well as a numerical description of the signs of defects determined by the measurement results and required for diagnosing the sentences and opinions of experts. To develop a prototype, the operating modes of the equipment and its current normative characteristics must be taken into account; for example, when diagnosing the flow path, it is necessary to use the normative characteristics obtained from the results of periodic tests. As a prototype for diagnosing a turbine control system, its characteristics obtained from tests and adjustments after repair can be employed. When numerically describing defect signs, it is necessary, for example, to describe such expressions as turbine rotor increased vibration, reduced vacuum in the condenser, various steam pressure along the threads, etc. The complexity of this procedure lies in the fact that deviations of the indicators can be caused not only by the error of the instruments or of specific measurement scheme, but also by different values of the significant deviation of the same indicator in different diagnostic tasks.

Let us study the features of adaptation of each type of algorithms developed for a particular equipment and a particular thermal power plant to a different type of equipment and other conditions (schemes) of its operation.

\section{Results}

Calculation algorithms, for example diagnostic algorithms for a control system, as a rule, can be built into an automatic process control system, an automatic control system, or in a turbine regulating system. Figure 1 , as an example, shows a block diagram of an algorithm for diagnosing a stem breakage in a turbine highpressure control valve. The diagnostic sign of this defect is based on the fact that when the valve is open, the pressure behind it rises above the pressure in the control stage chamber. The diagnostic task is implemented as follows (for the 1st control valve as an example):

- the pressure in the control stage is compared on the adder with the pressure behind the 1st valve and the difference is applied to a threshold element that generates a discrete output signal at a predetermined difference value (for example, less than $0.5 \mathrm{MPa}$ );

- similarly, the valve control signal is compared on the adder with the value corresponding to the start of the 1 st valve opening $(5 \%$, as it is conventionally presumed 


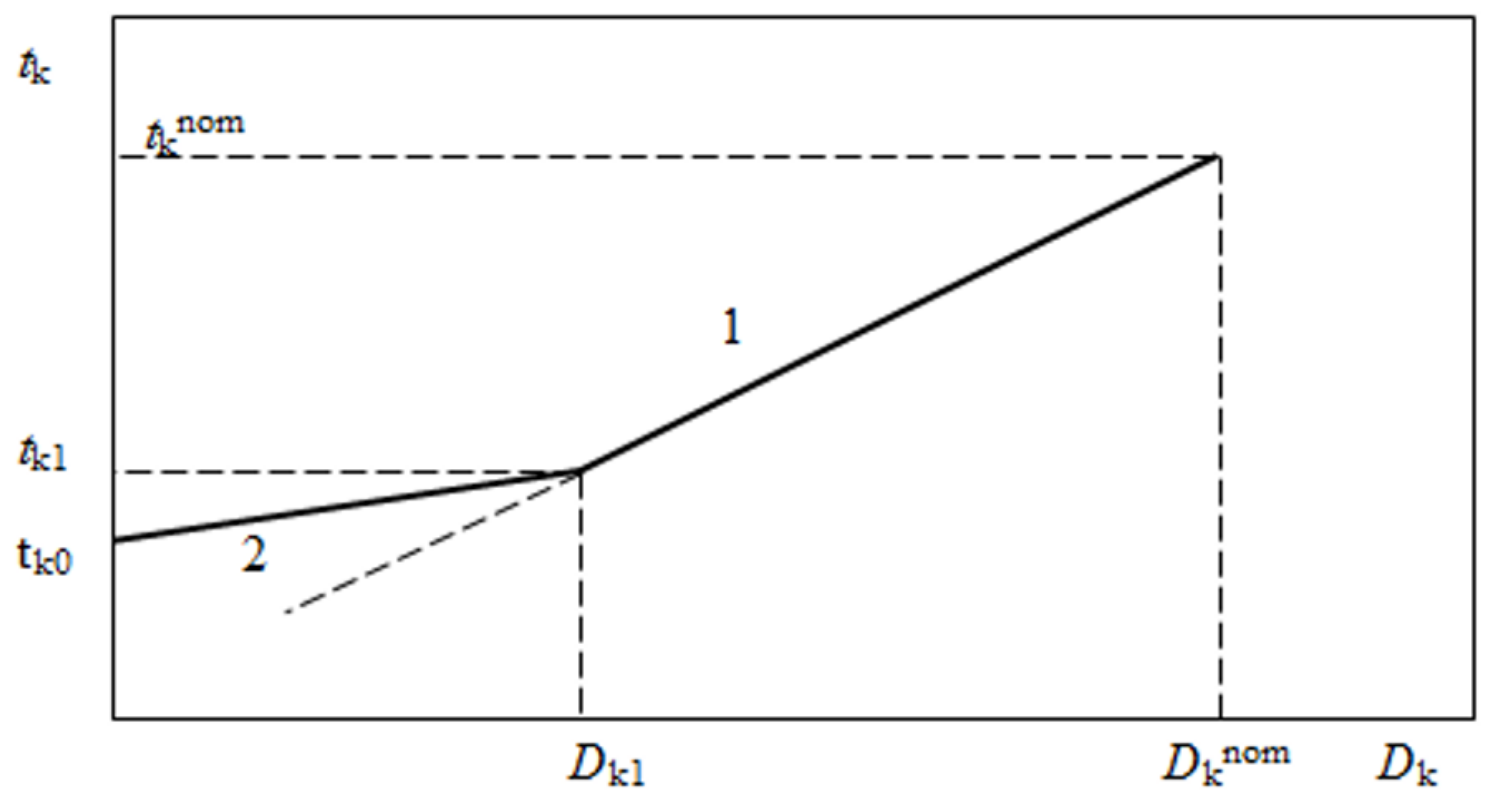

Fig. 2. Joint performance of the condenser and ejector: 1 - condenser performance without taking the ejector into account; $2-$ joint condenser-ejector performance.

in Figure 1) and the difference is applied to a threshold element that generates a discrete output signal with a predetermined difference, for example, more than $10 \%$ (this means the unconditional opening of the 1st valve);

- the considered diagnosis of valve stem breakage is possible if there is steam flow to the turbine, while the turbine is operating under load; to do this, the current generator power value is compared on the adder with the minimum accepted value (for example, $5 \%$ of the rated power of the turbine), the difference is applied to a threshold element that generates a discrete output signal when it is positive;

- if all three of the indicated discrete signals are supplied to the "AND" logic element, then a discrete signal is generated at the output of the latter, indicating a possible valve stem break.

Algorithms of this type are developed for a specific design of the regulatory system; the functioning of these algorithms clearly depends on the availability of the necessary measurements (and usually requires the inclusion of additional sensors in the traditional volume, steam pressure sensors behind the valves in particular). When adapting algorithms for a different type turbine or the same turbine of another thermal power plant, but with the same type of steam distribution, it is necessary to adjust the settings of the calculation algorithm (moments in valve opening, pressure difference values, etc.), i.e., to change the description (formalization) of diagnostic signs. Moreover, a complete revision of the algorithms (calculation model) is not required. For algorithms of this type, the presented diagnosis almost uniquely determines the defect that has appeared. With another type of turbine steam distribution (throttle type), a complete revision of the diagnostic algorithm (model) is required.

Fig. 2 presents the diagnostic model, on the basis of which a postoperative calculation algorithm is developed for assessing the separate effect of increased air suction and tube contamination on turbine condenser steam pressure. This problem should be solved when the actual steam pressure in the condenser is above the standard value; the condition of the main ejectors of the condensing unit is satisfactory, the ejector performance corresponds to the rating characteristic. The minimum frequency for the task to be launched for a solution is once in 7-10 days [11]. The algorithm for solving this problem is based on the construction of a joint performance of the condenser and ejector. The joint condenser-ejector performance (the dependence of the vapor saturation temperature, $t_{k}$, on the steam flow rate in the condenser) consists of two sections, as shown in Fig. 2 [12]. Section 1 corresponds to the condenser performance at high steam flow rates into the condenser from $D_{k 1}$ to $D_{k}{ }^{\text {nom }}$, where $D_{k}{ }^{\text {nom }}$ is the nominal steam flow rate, and $D_{k 1}=(0.4 \ldots 0.6) D_{k}{ }^{\text {nom }}$. In this range of $D_{k}$ variation, the influence of the ejector at standard air suction does not affect the efficiency of the condenser; steam temperature and, accordingly, steam pressure are determined by the standard performance. Section 2 presents a joint condenser-ejector performance and describes the efficiency of the condenser at low steam flow rates. Sections 1 and 2 are linear functions.

Assuming that the steam pressure deviation in the condenser from the standard value is the sum of two pressure differences determined by increased air suction $\left(\Delta \mathrm{P}_{\text {air }}\right)$ and by tube pollution $\left(\Delta \mathrm{P}_{\text {pol }}\right) \Delta \mathrm{P}_{\mathrm{k}}=\Delta \mathrm{P}_{\text {air }}+\Delta \mathrm{P}_{\text {pol }}$, so according to the calculation model [12], $\Delta \mathrm{P}_{\text {air }}$ and then $\Delta \mathrm{P}_{\mathrm{pol}}$ are found.

When adapting this algorithm for a turbine of a different type and size, it is necessary to employ the regulatory characteristics of specific equipment, and depending on the specific measurement scheme, revise the algorithms for diagnostic signs evaluating, that is, change the procedures of their formalization. The solution to this problem for a cogeneration turbine equipped with a condenser having an integrated tube 


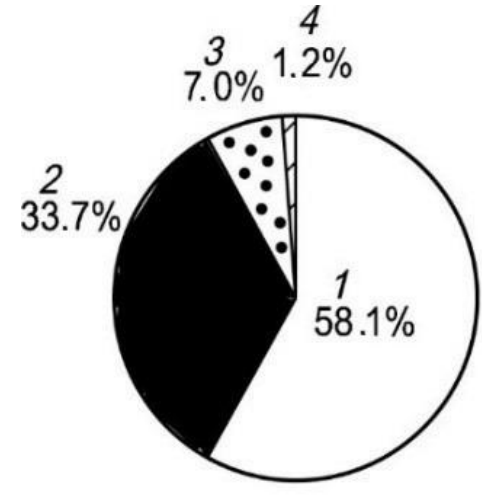

a

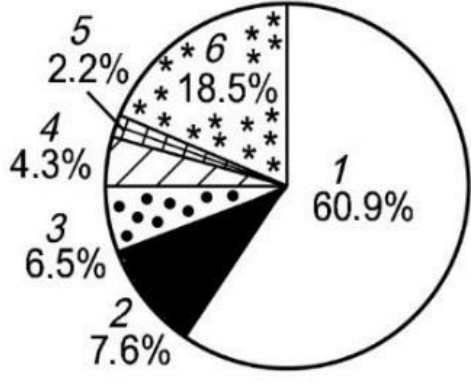

$\mathrm{b}$

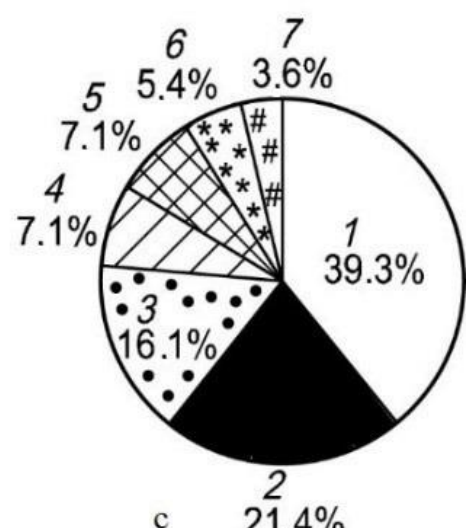

c $\quad 21.4 \%$

Fig. 3. Condenser damage distribution: a - distribution of condenser failure indications: 1 - increased condensate hardness, 2 - vacuum drop, 3 - si-phon breakdown, 4 - other; $b$ - condenser failure defect distribution: 1 - condenser tube damage, 2 — valve damage, drain damage at al., 3 - tubes and tube sheets pollution, 4 - condenser housing defects, 5 - loss of tightness of damaged tubes plugs, 6 - other; $\mathrm{c}$ - damage causes distribution of the condenser tube systems: 1 - frictional wear of tubes or rolled joints of tubes with tube plate caused by various reasons, 2 - tube erosion, 3 - tube corrosion, 4 - poor quality of brass tubes, $5-$ loss of zinc of the brass tubes, 6 - poor quality of rolling and of tube sheets during repair or manufacturing, 7 - tube breakage caused by blade fragments.

bundle, or for a condenser with a minimum steam consumption (when cogeneration diaphragm is completely closed) requires a change in the calculation model and a complete alteration of the algorithms.

Diagnostic algorithms of the first calculated (model) type are designed to identify specific defects and, in this aspect, provide maximum certainty of the analyzed defect.

The second type of algorithm (the expert one) presents the diagnosis of the presence of a defect in the form of probability. An algorithm of this type performs a procedure for assessing the presence or absence of signs characterizing a particular defect. The probability of a defect is calculated either as the sum of the weighting coefficients of the features present, or as a posteriori probability in the case of a Bayesian expert system.

The most important procedures for adapting these algorithms to various kinds of equipment are the analysis of the specific equipment damage at a specific thermal power plant and the formalization of expert knowledge regarding the assessment of a sign. An analysis of the damageability of equipment is of great importance, in particular, in determining the weight coefficients and probabilities of signs and a priori probabilities of hypotheses. As an example, Fig. 3 shows data on characteristic defects, the causes of defects and signs of their manifestation for turbine condensers, on the basis of which data the values of these probabilities are determined by the experts.

If the design of this equipment involves typical (previously used on other equipment) structural elements, it is advisable for diagnosis reliability increasing to evaluate (clarify) the probability of defects and signs occurrence based on statistics of their damageability.

Thus, to assess the probabilities of signs and defects when adapting expert-type diagnostic algorithms, one needs to formalize the knowledge of experts regarding a specific measurement scheme and design features of the equipment. The volume of equipment with measuring instruments is not always sufficient. In particular, when diagnosing a thermal expansion system, it is necessary to measure the following values [13]:

- absolute displacements of bearing housings to the left and right of the turbine axis,

- bevel slopes under bearing housings to the left and right of the turbine axis,

- lateral displacements of cylinder legs relative to bearing housings.

In the current industrial control systems, the absolute displacements of the bearing housings and the bevel slopes under the bearing housings are usually measured on one side, and the transverse displacements of the cylinder legs are not determined. It is necessary for each parameter used in the diagnosis to assign a value corresponding to its significant change. Moreover, a change in one and the same physical parameter in different tasks can be different. Thus, a significant temperature change for diagnosing thermal expansion system (a change in the temperature of the cylinder metal) that affects the cylinder displacement is $50{ }^{\circ} \mathrm{C}$, while the circulation water temperature change for diagnosing the condensation unit is $0.5 \ldots 1.0^{\circ} \mathrm{C}$. In this case, the error of the device plays an important role; in automated control systems the aperture of addition to the database can be $2 / 3$ of the error of the device.

The signs of defects encountered in the literature are formulated in a general way, and when developing algorithms for a specific unit, it is necessary to take into account its specific features: the number of bearings; the availability of steam extractions for industrial and heating purposes, their location relative to the turbine supports; the geometric characteristics of the cylinders, key joints, the possible values of the mutual displacement of the elements; the results of the thermal calculation determine the features of the algorithms for evaluating vibration signs and malfunctions, as well as a faulty system of turbine thermal expansions. 
For example, the diagnostic sign of "vibration dependence on the operating mode" should be formalized for a specific unit with regard to its design and scheme for parameter measuring. The material of condensers tubes, and of low pressure feed water heaters, hot water heaters, the presence of an integrated tube bundle in the condenser, the number and type of heaters in the system of regenerative feed water heating must be taken into account when determining the list of diagnostic tasks for auxiliary equipment.

As an example, in [11] a diagnostic sign of misalignment of the steam turbine rotors is presented as "a change in vibration with a change in electric or thermal load and condenser pressure". To create an algorithm for processing this feature, it is necessary to determine for each of the specified parameters a value, in case of exceeding which a change in the parameter is accepted. In addition, it is necessary to decide by what parameter the change in the heat load is estimated: by the pressure in the hot water heating extraction, by the opening of the diaphragm or the calculated parameter of the heat load, and if the turbine has several extractions, it is necessary to determine which rotor will be affected by the load change of each cogeneration extraction. For each parameter, it is necessary to describe a criterion a limiting value at which it can be considered that the sign is present.

Thus, for expert-type algorithms, the adaptation of the diagnostic system to another type of turbine may consist in the adjustment of sign evaluation algorithms. When transferring these algorithms from one type of turbine to another, the list of defects, signs, and also algorithms for sign evaluating (formalization of signs) may change; however, the algorithm for calculating the probability of a defect remains unchanged. If there are not enough sensors and corresponding measurements to evaluate a particular sign, then the degree of certainty of the defect assessment (but not the probability, if the missing measurements are replaced by the expert answers) is reduced. In the ultimate case, that is in the absence of measurements (and expert answers), the probability of a defect coincides with its a priori probability for a system constructed by the Bayes theorem. Expert systems based on rigid logic, such as a "decision tree", determine an unambiguous relationship between signs and defects. Such algorithms, which are expert in their form (developed by experts), in essence can be attributed to the calculation type, where the degree of certainty of the diagnosed defect is high.

The third type of algorithms - the digital one involves the equipment malfunctions diagnosing by comparing the actual values of the process parameters with the model process generated on the basis of the data from the automatic process control system based on the results of a rather long-term operation of the turbine unit [14]. This approach requires minimal effort to adapt the algorithms developed for turbine unit of one type to another. However, in this case, the degree of certainty in the assessment of a specific defect is significantly reduced. So, during the operation of such a system at one of the thermal power plants, the staff received a notification about the defect of the turbine unit - a level decrease in the in the oil tank, which was caused by routine work due to the oil replacement in the turbine oil system. With digital algorithms even the participation of experts in the assessment of defects does not guarantee an increase in certainty in finding defects.

\section{Discussion}

Most of the diagnostic approaches [15-20] use the prescriptive documents of Russia Federation, equipment passport performances and results of regulated (periodic) tests. Expert systems are based on the huge volume of data analyzed. For particular diagnostic tasks, additional methods of results validation are used. For example, for calculations with a full turbine scheme including the heat-mass transfer, balances are being made up. All these parts of the diagnostic process allow us to accept the accuracy of the results.

\section{Conclusions}

Diagnostic features for steam turbine equipment is determined by wide range of various equipment and diagnostic tasks. In addition, each turbine is operating in various conditions and has design differences even comparing to same series turbines.

Authors designed algorithms for range of defects diagnostics and for estimation of state of steam turbine equipment: flow part efficiency; vibrational defects (over 30 ), heat expansion system defects (3 defects groups), regulation system (10 diagnostic tasks) and auxiliary equipment, which includes condensers, boilers, ejectors, etc. (15 diagnostic tasks). Some diagnostic tasks are operating on-line, others - in postoperative mode. Diagnostic tasks are intended for defects revealing and equipment state predicting.

Defects determination is embodied in various algorithm types: calculating, expert, digital types. Principles of algorithms adaptation to specific turbines and operating conditions are designed by authors. Adaptation is also provided for a range of new turbines. Some algorithms are already operating at thermal power plants for more than 10 years.

\section{References}

[1] I.A. Perminov, V.G. Orlik, Diagnostics of the technical condition of the flow path of high and medium pressure cylinders by operational measurements of steam pressure and temperature in the turbine, Electric Power Stations 6, 3841(2003)

[2] S.Sh. Rosenberg, L.A. Khomenok, A.A. Gordinsky, V.I. Ilyin, Diagnostics of the condition of radial clearances in the cylinder flow path during operation, CKTI Transactions 273, 67-76 (1992)

[3] I.A. Kovalev, Development of algorithms for the functioning and recognition of defects for an 
automatic vibration diagnostic system, CKTI Transactions 273, 27-33 (1992)

[4] A.Z. Zile, M.N. Rudenko, S.B. Tomashevsky, V.G. Vasilyev, I.S. Khramov, The experience in implementing a system of vibrodiagnostic control of turbine units, Power Engineer 3, 21-23 (1999)

[5] A.Z. Zile, A.A. Romashev, S.A. Lymar, et al., Automated system for vibration control and diagnostics of a turbine unit of T-250/300-240 type, Electric Power Stations 3, 23-26 (1987)

[6] E.A. Don, D.V. Taraday, K.E. Buglaev, Diagnostic system for temperature expansion of turbine units, Electric Power Stations 9, 42-47 (2012)

[7] L.S. Baran, Development of a system for comprehensive technical diagnostics of a condensing unit for a turbine of K-800-240-3 type, CKTI Transactions 279, 40-51 (1994)

[8] Yu.M. Brodov, K.E. Aronson, M.A. Nierenstein, Steam turbine condensing unit diagnostic system concept. Thermal Engineering 7, 34-38 (1997)

[9] A.D. Trukhniy, N.A. Zroychikov, B.V. Lomakin, I.V. Sedov, Information and diagnostic system for monitoring hot water heaters for turbine of $\mathrm{T}$ 250/300-240 type, Thermal Engineering 1, 30-34 (1998)

[10] K.E. Aronson, B.E. Murmansky, Yu.M. Brodov, V.B. Novoselov, A.Yu. Sosnovsky, I.B. Murmansky, Forming of prototypes and features for diagnostics automation of steam turbine unit equipment. Thermal Engineering (2020)

[11] Guidelines for steam turbine condensing units testing: $\quad M U$ 34-70-010-82 (M.: $\quad$ SPO Soyuztechenergo, 68, 1982)

[12] S.I. Khaet, K.E. Aronson, Yu.M. Brodov, A.G. Shempelev, Development and testing of system elements for monitoring the condition and diagnostics of a steam turbine condenser, Thermal Engineering 7, 67-69 (2003)

[13] B.E. Murmansky, A.Yu. Sosnovsky, Yu.M. Brodov, Development of a module for monitoring and diagnostics of the system of steam turbine thermal expansions as part of modern automated control systems, Power Engineer 4, 51-53 (2015)

[14] E.V. Uriev, Fundamentals of reliability and technical diagnostics of turbomachinery: tutorial (Ekaterinburg: USTU, 71, 1996)

[15] A. Brooking, P. Johns, F. Cox, ed. R. Forsyth, Expert Systems, Principles and case studies (M.: Radio \& Communication, 191, 1987)
[16] Yu.M. Brodov, K.E. Aronson, B.E. Murmanskiy, I.B. Murmanskii, M.A. Nirenshtein, V.B. Novoselov, P.N. Plotnikov, A.Yu. Ryabchikov, Reliability of the equipment of steam turbine plants: a training manual (Ekaterinburg: Publishing House of the Ural Federal University, 144, 2017)

[17] Yu.M. Brodov, K.E. Aronson, A.Yu. Ryabchikov, M.A. Nirenstein, N.V. Zhelonkin, D.V. Brezgin, I.B. Murmanskii, The current situation and trends in the design and operation of condensers of powerful steam turbines: a Training manual (Ekaterinburg, publishing house of the Ural University, 104, 2019)

[18] Yu.M. Brodov, K.E. Aronson, A.Yu. Ryabchikov, M.A. Nirenshtein, I.B. Murmanskii, N.V. Zhelonkin, The current level and trends in the design and operation of heaters for the regeneration system of steam turbines of TPPs and NPPs: textbook (Yekaterinburg, Publishing House of the Ural University, 100, 2019)

[19] K.E. Aronson, S.N. Blinkov, V.I. Brezgin, Yu.M. Brodov, V.K. Kuptsov, I.D. Larionov, et al., Heat exchangers of power plants: textbook for universities, third edition, revised and supplemented (Yekaterinburg: UrFU, 828, 2014)

[20] Yu.M. Brodov, K.E. Aronson, Yu.M. Gofman, B.E. Murmansky, M.A. Nirenstein, A.Yu. Ryabchikov, P.N. Plotnikov, Repair and maintenance of steam turbine equipment, reference book in 2 volumes (Yekaterinburg, Publishing House of the Ural Federal University, 100, 2019) 